\title{
Most Malignant Fibrous Histiocytomas Developed in the Retroperitoneum Are Dedifferentiated Liposarcomas: A Review of 25 Cases Initially Diagnosed as Malignant Fibrous Histiocytoma
}

Jean-Michel Coindre, M.D., Odette Mariani, M.Sc., Frédéric Chibon, Ph.D., Aline Mairal, M.Sc., Nicolas de Saint Aubain Somerhausen, M.D., Elizabeth Favre-Guillevin, M.D., Nguyen Binh Bui, M.D., Eberhard Stoeckle, M.D., Isabelle Hostein, Ph.D., Alain Aurias, M.D.

Department of Pathology (JMC, IH), Medical Oncology (EFG, NBB) and Surgery (ES), Institut Bergonié, Bordeaux, France; Laboratoire de Pathologie Moléculaire des Cancers, INSERM U 509 (OM, FC, AM, AA), Institut Curie, Paris, France; and Department of Pathology (NSAS), Institut Jules Bordet, Université Libre de Bruxelles, Brussels, Belgium

Forty-four samples from 25 cases of retroperitoneal sarcoma initially diagnosed as malignant fibrous histiocytoma were histologically reviewed. Immunohistochemistry for $\mathrm{mdm} 2$ and cdk4 was performed on 20 cases. Comparative genomic hybridization was performed on 18 samples from 13 patients. Seventeen cases were reclassified as dedifferentiated liposarcoma. Twenty-one of 32 samples from these patients showed areas of well-differentiated liposarcoma, allowing the diagnosis of dedifferentiated liposarcoma. Immunohistochemistry performed in 15 of these cases showed positivity for mdm2 and cdk4. Comparative genomic hybridization analysis performed on 15 samples from 11 of these patients showed an amplification of the 12q13-15 region. Eight cases were reclassified as poorly differentiated sarcoma. Twelve samples from these patients showed no area of well-differentiated liposarcoma. Immunohistochemistry showed positivity for $\mathrm{mdm} 2$ and cdk4 in one of six of these patients and showed positivity for CD34 in another one. Comparative genomic hybridization analysis performed on three samples from two of these patients showed no amplification of the 12q13-15 region but showed complex profiles. This study shows that most so-called malignant fibrous

\footnotetext{
Copyright ( $\odot 2003$ by The United States and Canadian Academy of Pathology, Inc.

VOL. 16, NO. 3, P. 256, 2003 Printed in the U.S.A.

Date of acceptance: January 8, 2003.

This work was supported by the Ligue Nationale Contre le Cancer, Committees of Charente-Maritime and Pyrénées Atlantiques.

Address reprint requests to: Jean-Michel Coindre, M.D., Département de Pathologie, Institut Bergonié, 229, Cours de l'Argonne, 33076 Bordeaux Cédex, France; fax: 556-33-04-38; e-mail: coindre@bergonie.org.

DOI: 10.1097/01.MP.0000056983.78547.77
}

histiocytomas developed in the retroperitoneum are dedifferentiated liposarcoma and that a poorly differentiated sarcoma in this area should prompt extensive sampling to demonstrate a welldifferentiated liposarcoma component, immunohistochemistry for mdm2 and cdk4, and if possible, a cytogenetic or a molecular biology analysis.

KEY WORDS: Cdk4, Comparative genomic hybridization, Dedifferentiated liposarcoma, Immunohistochemistry, Malignant fibrous histiocytoma, Mdm2, Retroperitoneum.

Mod Pathol 2003;16(3):256-262

Malignant fibrous histiocytoma (MFH) was first described as a distinct histologic type of soft tissue sarcomas in 1964 (1), and thereafter, large series of cases were reported (2-4). For several years, MFH has been considered the most common soft tissue sarcoma of adult patients (5-7). A few years ago, Fletcher (8) doubted whether MFH was a diagnostic entity, and he emphasized that in most cases initially diagnosed as so-called MFH, a specific line of differentiation can be demonstrated, so only a few cases may be classified as undifferentiated pleomorphic sarcoma.

Retroperitoneal sarcomas represent between 10 and $15 \%$ of all soft tissue sarcomas in adults $(9,10)$. The most frequent type encountered in this location is liposarcoma, well-differentiated or dedifferentiated types, followed by leiomyosarcoma and MFH. Dedifferentiated liposarcomas mainly occur in the retroperitoneal space, and the most common pattern of dedifferentiated areas consists of highgrade pleomorphic MFH or storiform fibroblastic MFH $(11,12)$. Nowadays, many pathologists recognize that most so-called MFH located in the retro- 
peritoneum are dedifferentiated liposarcomas. In a recent study, we reported that a subgroup of MFH was associated with a specific genetic pattern similar to that of dedifferentiated liposarcomas (13), particularly when they are located in the retroperitoneum. However, no histologic study has been published so far, so we reviewed 25 cases of retroperitoneal sarcomas initially diagnosed as $\mathrm{MFH}$, fibrosarcoma, or undifferentiated sarcoma. We considered all tumoral events of patients treated and followed up in our center. Moreover, comparative genomic hybridization (CGH) was performed in 13 cases.

\section{MATERIALS AND METHODS}

From 1984 to 2000, 124 patients with a retroperitoneal sarcoma were referred to our center for the treatment of the primary tumor or the first local recurrence. These sarcomas were 58 liposarcomas (21 well-differentiated liposarcomas and 37 dedifferentiated liposarcomas); 25 sarcomas initially diagnosed as MFH, fibrosarcoma, or undifferentiated sarcoma; 22 leiomyosarcomas; and 19 other sarcomas. The group of sarcomas initially diagnosed as $\mathrm{MFH}$, fibrosarcomas, or undifferentiated sarcoma is the subject of this study. There were 16 male patients and 9 female patients; patient ages ranged from 37 to 86 years (median age, $62 \mathrm{y}$ ).

Histologic review was performed on all resected tumoral events, that is, 44 samples: 25 primary tumors before treatment (21 tumor resections and 4 open biopsies), 4 resections after chemotherapy, 13 local recurrences, 1 metastasis, and 1 persistent disease. A total of 1 to 60 paraffin blocks (median, 15) were performed on the samples with 1 to 41 blocks (median, 10) containing tumor tissue.

Immunohistochemistry was performed on selected cases on a representative paraffin block. The following antibodies were used: cytokeratin (Kl1, Immunotech), EMA (E29, DAKO), S-100 protein (polyclonal, DAKO), desmin (D33, DAKO), alpha-smooth muscle actin (IA4, Sigma), myogenin (LO26, Novocastra), h-caldesmon (H-CD, DAKO), mdm2 (IF2, Zymed), and cdk4 (DCS-31, Biosource International). Immunostaining was performed according to the streptavidin-biotin-peroxidase method of Hsu et al. (14). Tissue sections were submitted to microwave oven heating ( $20 \mathrm{~min}$ in $0.1 \mathrm{M}$ citrate buffer at $\mathrm{pH} 6$ ) or to trypsin digestion $(0.1 \%$ in $0.2 \% \mathrm{CaCl}$ solution, 10 $\min$ at $37^{\circ} \mathrm{C}$ ) before staining. Then, the sections were immunostained using the LSAB kit (DAKO) in an automated immunostainer (DAKO TechMate-TM Horizon, DAKO Denmark). All steps were performed at room temperature, and diaminobenzidine (DAKOpatts) was used as a chromogen. Appropriate positive and negative controls were employed throughout.
Chromosomal CGH was performed on frozen tissue obtained in 18 samples from 13 patients, according to the method described elsewhere (15)

Dedifferentiated liposarcoma was diagnosed on histologic criteria. It was defined by the presence of a clear-cut, well-differentiated liposarcoma component clearly separated from the poorly differentiated component and/or occupying a large area beside the poorly differentiated component. Identification of the well-differentiated component depended on the presence either of significant nuclear atypia or pleomorphism in a fatty component or of lipoblasts. All cases were reviewed independently by two of the authors (JMC and NSAS), and a well-differentiated liposarcoma component was retained only when both reviewers agreed.

\section{RESULTS}

After careful histologic review of all tumoral events, immunohistochemical data, and CGH profile of some cases, revised diagnoses were dedifferentiated liposarcoma for 17 cases and poorly differentiated sarcomas for 8 cases, 1 being consistent with a malignant solitary fibrous tumor (Table).

\section{Dedifferentiated Liposarcomas}

Seventeen cases with 32 samples were reclassified as dedifferentiated liposarcoma and showed histologic areas of well-differentiated liposarcoma on at least one tumoral event.

In 11 cases, this well-differentiated liposarcoma component was seen on the primary tumor resection before any treatment. Five of these patients experienced a local recurrence with a well-differentiated liposarcoma component in three cases and no welldifferentiated liposarcoma component in two cases (Cases 5, with one local recurrence, and 13, with two local recurrences). One patient (Case 9) had a distant metastasis that showed no well-differentiated liposarcoma component.

In three cases, a well-differentiated liposarcoma component was seen on the tumor resection after chemotherapy, whereas the initial biopsy showed no well-differentiated liposarcoma component. Only 2, 1 , and 4 paraffin blocks were performed on the biopsy before chemotherapy, whereas 5,33 , and 60 blocks were available on the tumor resection after chemotherapy for these 3 cases. For one patient (Case 12), a persistent disease was resected and showed no welldifferentiated liposarcoma component. Case 23 had a local recurrence that showed a well-differentiated liposarcoma component.

In three cases, a well-differentiated liposarcoma component was seen on the local recurrence but 
TABLE 1. Clinicopathological Features, Comparative Genomic Hybridization Results and Final Diagnosis of Cases in This Study

\begin{tabular}{|c|c|c|c|c|c|c|c|c|}
\hline Case Number & Age/sex & Year & Event & $\begin{array}{l}\text { Size } \\
(\mathrm{cm})\end{array}$ & $\begin{array}{l}\text { Number of } \\
\text { Blocks* }\end{array}$ & $\begin{array}{l}\text { Presence of } \\
\text { WDLS* }\end{array}$ & $\mathrm{CGH}^{*}$ & Final Diagnosis \\
\hline 1 & $69 / \mathrm{F}$ & 1984 & Primary & 10 & $4(3)$ & No & nd & PDS/MFH* \\
\hline 2 & $40 / \mathrm{M}$ & 1984 & Primary & 8 & $3(3)$ & No & nd & PDS/MFH \\
\hline \multirow[t]{2}{*}{3} & $37 / \mathrm{F}$ & 1985 & Primary & 12 & $2(2)$ & No & nd & DDLS* \\
\hline & & 1995 & LR & 35 & $37(37)$ & Yes (100\%) & + & \\
\hline \multirow[t]{2}{*}{4} & $62 / \mathrm{F}$ & 1986 & Primary & 20 & $17(13)$ & Yes $(20 \%)$ & nd & DDLS \\
\hline & & 2001 & LR & 23 & $50(33)$ & Yes $(95 \%)$ & + & \\
\hline \multirow[t]{2}{*}{5} & $66 / \mathrm{M}$ & 1986 & Primary & 12 & $16(14)$ & Yes $(30 \%)$ & nd & DDLS \\
\hline & & 1991 & LR & 19 & $19(18)$ & No & + & \\
\hline \multirow[t]{2}{*}{6} & $64 / \mathrm{M}$ & 1986 & Primary & 15 & $4(4)$ & Yes $(10 \%)$ & nd & DDLS \\
\hline & & 1986 & LR & $?$ & $35(9)$ & Yes $(30 \%)$ & nd & \\
\hline 7 & $72 / \mathrm{M}$ & 1986 & Primary & 18 & $6(6)$ & No & nd & PDS/MFH \\
\hline 8 & $60 / \mathrm{M}$ & 1987 & Primary & 20 & $11(7)$ & Yes $(10 \%)$ & nd & DDLS \\
\hline \multirow{2}{*}{9} & $73 / \mathrm{F}$ & 1987 & Primary & 10 & 17 (16) & Yes $(40 \%)$ & nd & DDLS \\
\hline & & 1989 & Meta & ? & $4(4)$ & No & nd & \\
\hline \multirow[t]{2}{*}{10} & $73 / \mathrm{M}$ & 1988 & Primary & 10 & $8(5)$ & No & nd & PDS/MFH \\
\hline & & 1989 & LR & 17 & $4(4)$ & No & nd & \\
\hline \multirow[t]{2}{*}{11} & $64 / \mathrm{M}$ & 1988 & Primary & 20 & $3(2)$ & No & nd & DDLS \\
\hline & & 1992 & LR & 15 & $58(33)$ & Yes $(50 \%)$ & + & \\
\hline \multirow[t]{3}{*}{12} & $47 / \mathrm{M}$ & 1989 & Primary (B) & 30 & $2(2)$ & No & nd & DDLS \\
\hline & & 1990 & Primary (PC) & 18 & $5(3)$ & Yes $(20 \%)$ & + & \\
\hline & & 1991 & Persistant D & 25 & $33(31)$ & No & + & \\
\hline \multirow[t]{3}{*}{13} & $58 / \mathrm{M}$ & 1989 & Primary & 16 & $35(20)$ & Yes $(20 \%)$ & + & DDLS \\
\hline & & 1995 & LR & 8 & $33(21)$ & No & + & \\
\hline & & 1997 & LR & 25 & $3(2)$ & No & nd & \\
\hline 14 & $58 / \mathrm{M}$ & 1991 & Primary & 10 & $4(4)$ & Yes $(20 \%)$ & nd & DDLS \\
\hline \multirow[t]{3}{*}{15} & $41 / \mathrm{M}$ & 1992 & Primary (B) & 23 & $3(3)$ & No & nd & $\mathrm{PDS} / \mathrm{MFH}$ \\
\hline & & 1992 & Primary (PC) & 11 & $25(16)$ & No & - & \\
\hline & & 1993 & LR & 9 & $29(14)$ & No & - & \\
\hline \multirow[t]{2}{*}{16} & $67 / \mathrm{F}$ & 1992 & Primary & 17 & 17 (17) & No & nd & PDS/MFH \\
\hline & & 1993 & LR & 15 & $15(13)$ & No & nd & \\
\hline 17 & $40 / \mathrm{F}$ & 1992 & Primary & 20 & $8(8)$ & Yes $(30 \%)$ & nd & DDLS \\
\hline \multirow[t]{2}{*}{18} & $86 / \mathrm{M}$ & 1992 & Primary & 18 & $1(1)$ & No & nd & DDLS \\
\hline & & 1993 & LR & 28 & 40 (35) & Yes $(<5 \%)$ & + & \\
\hline \multirow[t]{2}{*}{19} & $61 / \mathrm{F}$ & 1993 & Primary & 15 & $49(24)$ & Yes $(60 \%)$ & + & DDLS \\
\hline & & 1997 & LR & 9 & 33 (33) & Yes $(95 \%)$ & + & \\
\hline 20 & $73 / \mathrm{F}$ & 1993 & Primary & 20 & $11(10)$ & No & - & PDS/MFH \\
\hline 21 & $50 / \mathrm{M}$ & 1994 & Primary & 16 & $4(4)$ & No & nd & PDS/SFT* \\
\hline 22 & $47 / \mathrm{M}$ & 1994 & Primary & 10 & $18(14)$ & Yes $(30 \%)$ & nd & DDLS \\
\hline \multirow[t]{3}{*}{23} & $72 / \mathrm{F}$ & 1995 & Primary (B) & 30 & $1(1)$ & No & nd & DDLS \\
\hline & & 1996 & Primary (PC) & 30 & 33 (13) & Yes $(70 \%)$ & + & \\
\hline & & 1999 & LR & 12 & $30(28)$ & Yes $(40 \%)$ & + & \\
\hline \multirow[t]{2}{*}{24} & $62 / \mathrm{M}$ & 1997 & Primary (B) & 20 & $4(4)$ & No & nd & DDLS \\
\hline & & 1998 & Primary (PC) & 12 & $60(41)$ & Yes $(70 \%)$ & + & \\
\hline 25 & $57 / \mathrm{M}$ & 2000 & Primary & 10 & $46(33)$ & Yes $(<5 \%)$ & + & DDLS \\
\hline
\end{tabular}

LR = local recurrence; $\mathrm{B}=$ biopsy; $\mathrm{PC}=$ postchemotherapy; $\mathrm{D}=$ disease; Number of blocks $=$ on the sample (containing tumor tissue); WDLS $=$ well-differentiated liposarcoma component; $\mathrm{CGH}=$ comparative genomic hybridization; $\mathrm{nd}=$ not done; $+=$ genomic profile of well-differentiated/ dedifferentiated liposarcoma; - = complex genomic profile imbalances suggesting a leiomyosarcoma; PDS/MFH $=$ poorly differentiated sarcoma/ malignant fibrous histiocytoma; DDLS = dedifferentiated liposarcoma; PDS/SFT = poorly differentiated sarcoma consistent with a malignant solitary fibrous tumor.

not on the primary tumor (Fig. 1). For these cases, only 2,3 , and 1 paraffin blocks were performed on the primary tumor, whereas 37,58 , and 40 blocks were studied on the local recurrence. In one of these cases (Case 18), the well-differentiated liposarcoma component was seen on only one slide.

A total of 32 samples was histologically reviewed: 21 with 4 to 60 paraffin blocks (median, 33 blocks) showed a well-differentiated liposarcoma component, and 11 with 1 to 33 paraffin blocks (median, 4 blocks) showed no well-differentiated liposarcoma component.

Immunohistochemistry was performed on 15 cases with anti-mdm2 and cdk4: every case showed tumor cell positivity for both antibodies.
CGH analysis was performed on 15 tumoral events from 11 patients and showed a typical amplification of the 12 q13-15 region in every case, with an amplification of $1 \mathrm{p} 32$ in four cases and of $6 q 32$ in one case.

For Cases 13 and 25, a diagnosis of dedifferentiated liposarcoma was considered on the basis of the CGH results. Case 13 was initially diagnosed as an inflammatory MFH, but review of slides showed a well-differentiated liposarcoma component representing about $20 \%$ of the whole tumor. This aspect was initially considered as an infiltration of the normal fat by tumor cells. Two local recurrences 6 and 8 years after the primary tumor were only composed of MFH areas. The first local recurrence was 

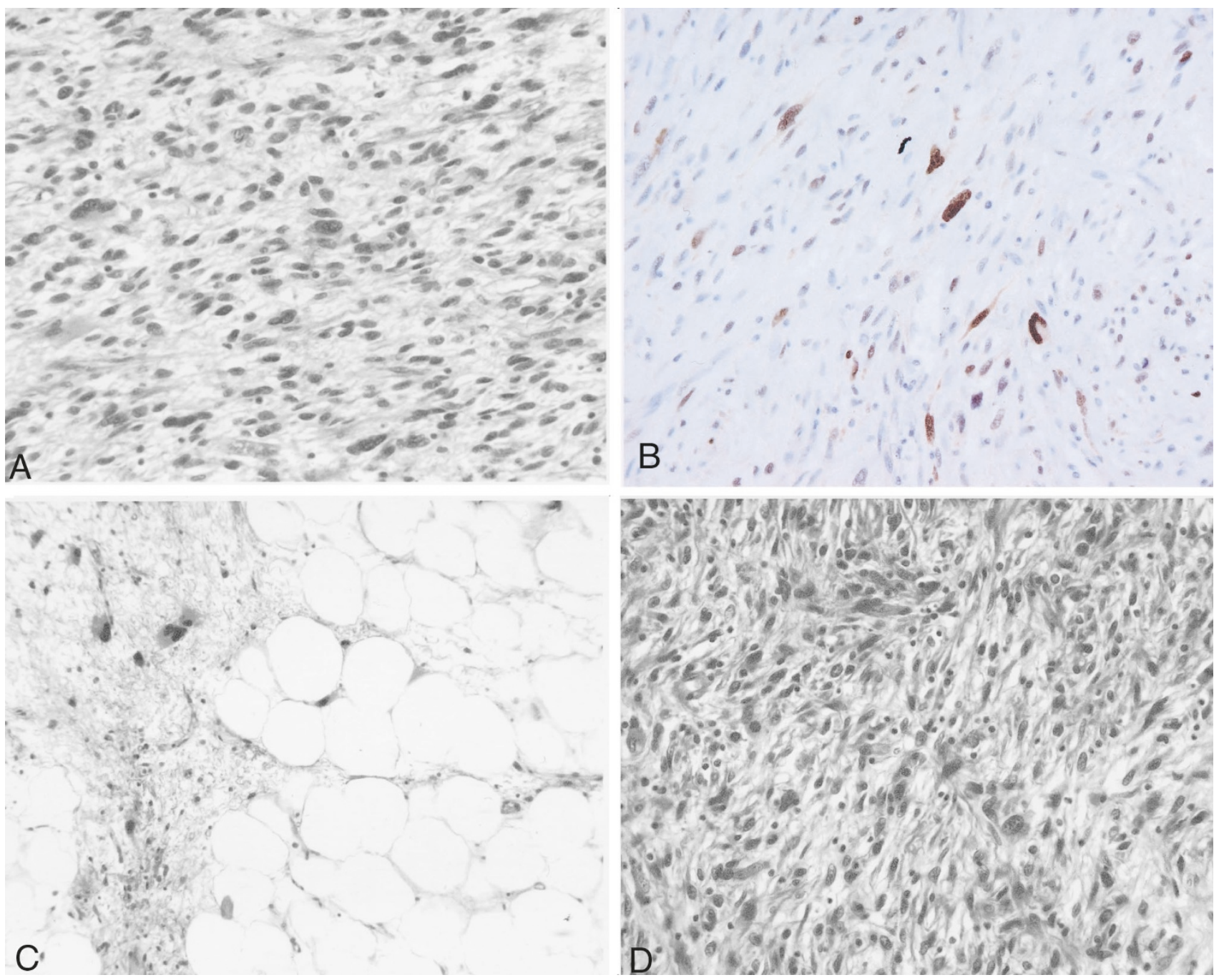

FIGURE 1. Case 11. Primary tumor: A, the primary tumor, composed of spindle and pleomorphic cells arranged in short fascicles, was diagnosed as an MFH (only 3 blocks were available); B, immunohistochemistry showed a strong positivity for anti-mdm2 on some tumor cells. Local recurrence (58 blocks): a local recurrence showed the typical aspect of a dedifferentiated liposarcoma combining areas of well-differentiated liposarcoma (C) and areas of poorly differentiated sarcoma (D).

also studied by CGH and showed the same genomic imbalances, with an amplification of the 12 q13-15 region. Case 25 was initially diagnosed as a pleomorphic MFH, and again CGH results suggested the diagnosis of dedifferentiated liposarcoma. Review of slides showed a small area of well-differentiated liposarcoma component, only on one slide (Fig. 2).

\section{Poorly Differentiated Sarcomas}

For 7 cases with 11 events, no well-differentiated liposarcoma component could be seen. These 11 events were studied on 3 to 29 blocks (median, 8 blocks). Histologically, these tumors were composed of spindle and/or pleomorphic cells arranged in short fascicles or without any pattern.

Immunohistochemistry was performed on five cases because for two cases, paraffin blocks were not available. The five studied cases were negative for cytokeratin, EMA, S100 protein, desmin, alpha smooth muscle actin, h-caldesmon, and myogenin. Four cases were also negative for $\mathrm{mdm} 2$ and cdk4, whereas the other one showed positive cells for these antibodies (Case 10).

CGH analysis was performed on three tumoral events from two cases (Cases 15 and 20). There was no amplification of 12q13-15, but complex CGH profiles similar to those described in leiomyosarcoma were observed.

One case (Case 21) was composed of round and ovoid cells intermingled with a few pleomorphic cells. CD34 and S100 protein were positive on about $50 \%$ of cells, but other markers (cytokeratin, EMA, desmin, alpha smooth muscle actin, mdm2, and cdk4) were negative. This case was reclassified as a poorly differentiated sarcoma consistent with a malignant solitary fibrous tumor. 

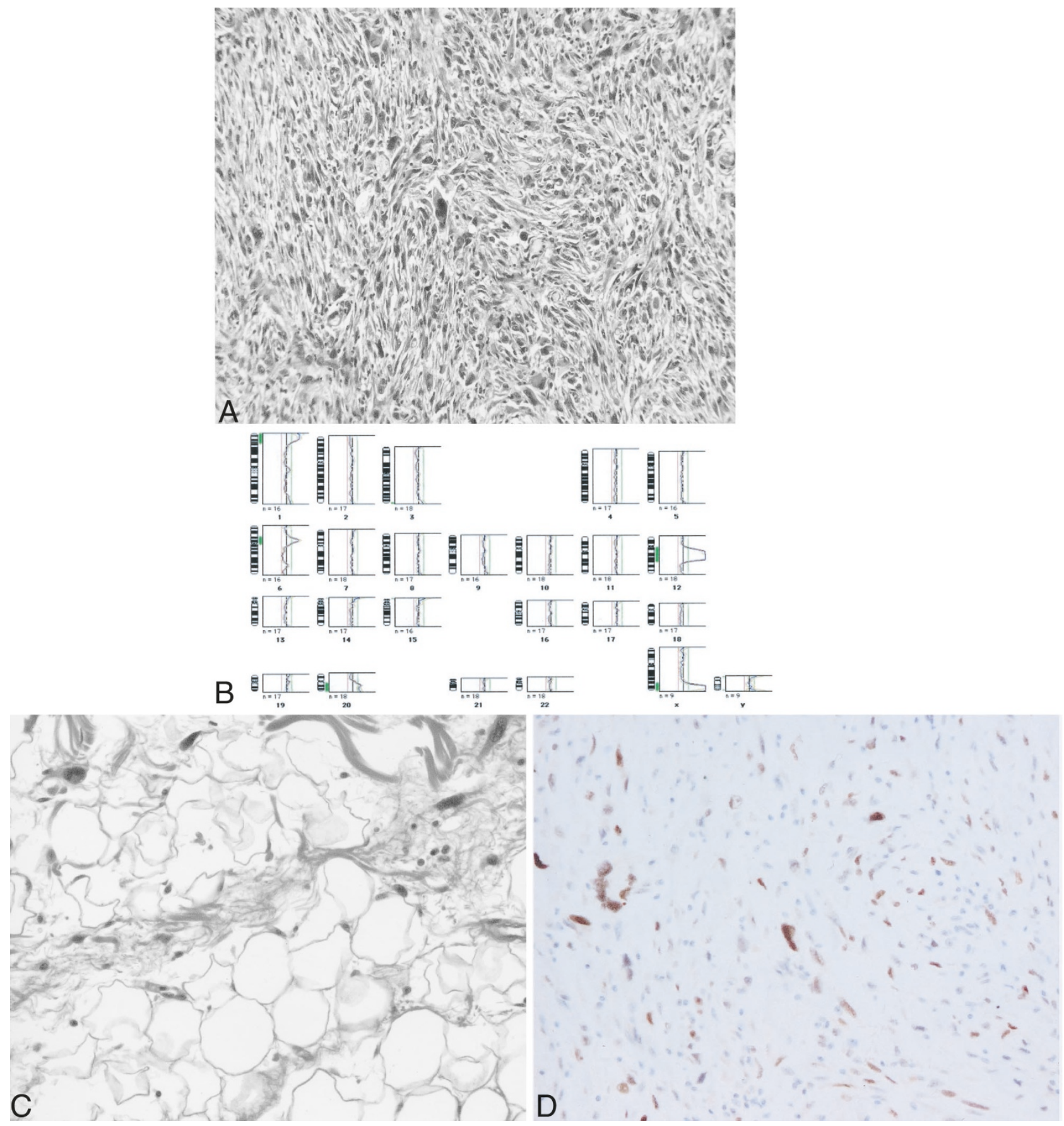

FIGURE 2. Case 25. A, most of the tumor was composed of pleomorphic and spindle cells, and initial diagnosis was MFH. B, comparative genomic hybridization showed an amplification of the 12q13-15 region, suggesting the diagnosis of dedifferentiated liposarcoma. C, review of the case showed a small area of well-differentiated liposarcoma on 1 of 46 slides. D, immunohistochemistry showed positivity of tumor cells for anti-mdm2.

\section{DISCUSSION}

The concept of MFH as a discrete entity is more and more controversial. Most tumors diagnosed as MFH are considered rather as poorly differentiated sarcomas for which a specific line of differentiation cannot be demonstrated because of technical limitations, such as in tumor sampling and technical investigations such as ultrastructural study, immunohistochemistry, and molecular analysis (8). They also could correspond to dedifferentiated sarcomas in which the well-differentiated component has not been involved by sampling or no longer exists. Therefore, MFH could represent a common morphologic appearance resulting from tumoral progression of various sarcomas, especially liposarcomas, but also others $(16,17)$.

In the retroperitoneal space, MFH represents 7 to $30 \%$ of sarcomas, and poorly differentiated sarcomas, that is, MFH, fibrosarcomas, malignant hemangiopericytomas and unclassified sarcomas, 
represent 16 to $50 \%$ of sarcomas, whereas liposarcomas represent 20 to $40 \%$ of sarcomas, and leiomyosarcomas, 10 to $30 \%$ of sarcomas $(10,18-$ 23). Retroperitoneal liposarcomas are differentiated or dedifferentiated types. Dedifferentiated areas usually consist of high-grade, poorly differentiated sarcomas resembling MFH or fibrosarcoma or, less often, of low-grade spindle cell or myxoid sarcomas resembling fibromatosis, well-differentiated fibrosarcoma, or myxofibrosarcoma. Divergent myxosarcomatous or osteosarcomatous differentiation can also be seen $(11,12)$. Dedifferentiated liposarcoma is diagnosed thanks to identification of areas of well-differentiated liposarcoma, most often of sclerosing subtype. Retroperitoneal liposarcomas are usually large tumors, and the proportion of well-differentiated liposarcoma and dedifferentiated liposarcoma components is variable. Therefore, extensive sampling is recommended to avoid missing any component. Sampling must be performed in both the nonadipose and adipose areas because the diagnosis of well-differentiated liposarcomas is regularly established in the latter. In our study, a well-differentiated liposarcoma component was identified in 21 of 32 samples from 17 patients. For the samples positive for welldifferentiated liposarcoma component, 4 to 60 blocks, with a median of 33 blocks, were available, whereas only 1 to 33 blocks with a median of 4 blocks were performed for the samples that were negative for well-differentiated liposarcoma component. This study also showed the importance of evaluating every tumoral event, as some may be entirely composed of dedifferentiated areas, whereas others are composed of both dedifferentiated liposarcoma and well-differentiated liposarcoma components or even of the welldifferentiated liposarcoma component only. In this series, 11 samples of 32 from the 17 patients with a dedifferentiated liposarcoma showed no welldifferentiated liposarcoma component at all.

Well-differentiated/dedifferentiated liposarcomas belong to the same category of liposarcomas characterized by ring or giant-marker chromosomes derived from the q13-15 region of chromosome 12, and amplification of this region results in amplification of mdm2, cdk4, SAS, and GLI genes $(24-26)$. In two previous studies $(13,27)$, we analyzed a series of 108 cases of so-called MFH by CGH and showed that most of them exhibited complex profiles of genomic imbalances similar to those described in leiomyosarcomas, suggesting that this subgroup of MFHs could correspond to dedifferentiated leiomyosarcomas. A second subgroup of 22 tumors showed a more simple CGH profile characterized by amplification of the 12q13-15 chromosome region, with additional amplifications of $1 \mathrm{p} 32$ or $6 q 23$. These results highly suggested that these tumors were dedifferentiated liposarcomas, especially as most of them were located in the retroperitoneum. The current study confirms the latter results by showing a perfect concordance between histologic features and CGH analysis. CGH analysis performed in 11 of 17 dedifferentiated liposarcomas showed the typical genomic profile of welldifferentiated/dedifferentiated liposarcomas. In 2 of 7 unclassified sarcomas, CGH analysis showed the complex genomic imbalances described in leiomyosarcomas. Genomic analysis has become more and more important in the diagnosis of soft tissue sarcomas, especially with the demonstration of specific reciprocal translocations in several sarcomas (28). Our studies suggest that the demonstration of amplifications and deletions could also be of interest for diagnosing leiomyosarcomas and particularly well-differentiated/dedifferentiated liposarcomas. In this series, a diagnosis of dedifferentiated liposarcoma was considered on the basis of the CGH results and was confirmed by histologic review in two patients (Cases 13 and 25). In three samples from three patients (Cases 5, 12, and 13), CGH analysis showed the typical genomic profile of well-differentiated/dedifferentiated liposarcoma, whereas histologic review showed no area of welldifferentiated liposarcoma. CGH analysis is a powerful tool for analyzing these abnormalities but is a time-consuming technique requiring considerable experience to obtain reproducible results. Therefore, it is not adapted to routine diagnosis. Amplifications of genes involved in the 12q13-15 region, particularly mdm2 and cdk4, may be demonstrated by quantitative PCR (29) or by immunohistochemistry $(30,31)$. In this series, immunohistochemical study showed a constant positivity of $\mathrm{mdm} 2$ and cdk4 in every dedifferentiated liposarcoma for which a paraffin block was available for immunohistochemistry. Four of five unclassified sarcomas were negative for these antibodies. One case (Case 10) was positive whereas no well-differentiated liposarcoma could be demonstrated, but only eight and four paraffin blocks were available on the two tumoral events for this case, and the immunohistochemical results may suggest that this tumor is a dedifferentiated liposarcoma. However, further studies on specificity and sensitivity of $\mathrm{mdm} 2$ and cdk4 should be performed because of the reports of positivity of these antibodies in other sarcomas and particularly in MFH (32-34). The question is whether these MFH are dedifferentiated liposarcomas or not.

In conclusion, a retroperitoneal poorly differentiated sarcoma resembling MFH or fibrosarcoma should prompt extensive sampling in order to demonstrate a well-differentiated liposarcoma component, immunohistochemistry for $\mathrm{mdm} 2$ and cdk4 and, if possible, a cytogenetic or a molecular biol- 
ogy study because it is probably a dedifferentiated liposarcoma.

\section{REFERENCES}

1. O'Brien JE, Stout AP. Malignant fibrous xanthomas. Cancer 1964;17:1445-55.

2. Weiss SW, Enzinger FM. Malignant fibrous histiocytoma. An analysis of 200 cases. Cancer 1978;4:2250-66.

3. Enjoji M, Hashimoto H, Tsuneyoshi M, Iwasaki H. Malignant fibrous histiocytoma. A clinicopathologic study of 130 cases. Acta Pathol Jpn 1980;30:727-41.

4. Kearney MM, Soule E, Ivins JC. Malignant fibrous histiocytoma. A retrospective study of 167 cases. Cancer 1980;45: 167-78.

5. Rooser B, Willen H, Gustafson P, Alvegard TA, Rydholm A. Malignant fibrous histiocytoma of soft tissue. A populationbased epidemiologic and prognostic study of 137 patients. Cancer 1991;15:499-505.

6. Enzinger FM. Malignant fibrous histiocytoma 20 years after Stout. Am J Surg Pathol 1986;10:43-53.

7. Le Doussal V, Coindre JM, Leroux A, Hacène K, Terrier P, Bui $\mathrm{NB}$, et al. Prognostic factors for patients with localized primary malignant fibrous histiocytoma: a multicenter study of 216 patients with multivariate analysis. Cancer 1996;77:1823-30.

8. Fletcher CDM. Pleomorphic malignant fibrous histiocytoma: fact or fiction? A critical reappraisal based on 159 tumors diagnosed as pleomorphic sarcoma. Am J Surg Pathol 1992; 16:213-28.

9. Heslin MJ, Lewis JJ, Nadler E, Newman E, Woodruff JM, Casper ES, et al. Prognostic factors associated with longterm survival for retroperitoneal sarcoma: implications for management. J Clin Oncol 1997;15:2832-9.

10. Stoeckle E, Coindre JM, Bonvalot S, Kantor G, Terrier Ph, Bonichon F, et al., from the French Federation of Cancer Centers Sarcoma Group. Prognostic factors in retroperitoneal sarcoma. A multivariate analysis of a series of 165 patients of the French Cancer Center Federation Sarcoma Group. Cancer 2001;92:359-68.

11. McCormick D, Mentzel T, Beham A, Fletcher CDM. Dedifferentiated liposarcoma. Clinicopathologic analysis of 32 cases suggesting a better prognostic subgroup among pleomorphic sarcomas. Am J Surg Pathol 1994;18:1213-23.

12. Henricks WH, Chu YC, Goldblum JR, Weiss SW. Dedifferentiated liposarcoma. A clinicopathologic analysis of 155 cases with a proposal for an expanded definition of dedifferentiation. Am J Surg Pathol 1997;21:271-81.

13. Chibon F, Mariani O, Derré J, Malinge S, Coindre JM, Guillou $\mathrm{L}$, et al. A subgroup of malignant fibrous histiocytomas is associated with specific genetic changes similar to those of well-differentiated liposarcomas. Cancer Genet Cytogenet 2002;139:24-9.

14. Hsu SM, Raine L, Fanger H. Use of avidin-biotin-peroxidase complex (ABC) in immunoperoxidase techniques: a comparison between $\mathrm{ABC}$ and unlabelled antibody (PAP) procedures. J Histochem Cytochem 1981;29:577-80.

15. Kallioniemi A, Kallioniemi OP, Sudar D, Rutovitz D, Gray JW, Waldman F, et al. Comparative genomic hybridization for molecular cytogenetic analysis of solid tumors. Science 1992; 258:818-21.

16. Brooks JJ. The significance of double phenotypic patterns and markers in human sarcomas. A new model of mesenchymal differentiation. Am J Pathol 1986;125:113-23.

17. Meis JM. "Dedifferentiation" in bone and soft-tissue tumors. A histological indicator of tumor progression. Pathol Annu 1991;26 Pt 1:37-62.
18. Pinson CW, ReMine SG, Fletcher WS, Braasch JW. Long-term results with primary retroperitoneal tumors. Arch Surg 1989; 124:1168-73.

19. Catton CN, O'Sullivan B, Kotwall C, Cummings B, Hao Y, Fornasier V. Outcome and prognosis in retroperitoneal soft tissue sarcoma. Int J Radiat Oncol Biol Phys 1994;29:1005-10.

20. Kilkenny JW, Bland KI, Copeland EM. Retroperitoneal sarcoma: the University of Florida experience. J Am Coll Surg 1996;182:329-39.

21. Karakousis CP, Velez AF, Gerstenbluth R, Driscoll DL. Resectability and survival in retroperitoneal sarcomas. Ann Surg Oncol 1996;3:150-8.

22. Lewis JJ, Leung D, Woodruff JM, Brennan MF. Retroperitoneal soft-tissue sarcoma. Analysis of 500 patients treated and followed at a single institution. Ann Surg 1998;228:355-65.

23. Herman K, Cruchala A, Niezabitowski A, Glinski B, Lackowska B. Prognostic factors in retroperitoneal sarcomas: ploidy of DNA as a predictor of clinical outcome. J Surg Oncol 1999;71:32-5.

24. Rosai J, Akerman M, Dal Cin P, DeWever I, Fletcher CDM, Mandahl N, et al. Combined morphologic and karyotypic study of 59 atypical lipomatous tumours. Evaluation of their relationship and differential diagnosis with other adipose tissue tumors (a report of the CHAMP Study Group). Am J Surg Pathol 1996;20:1182-9.

25. Rubin BP, Dal Cin P. The genetics of lipomatous tumors. Semin Diagn Pathol 2001;18:286-93.

26. Pédeutour F, Forus A, Coindre JM, Berner JM, Nicolo G, Michiels JF, et al. Structure of the supernumerary ring and giant rod chromosomes in adipose tissue tumors. Genes Chromosomes Cancer 1999;24:30-41.

27. Derré J, Lagacé R, Nicolas A, Mairal A, Chibon F, Coindre JM, et al. Leiomyosarcomas and most malignant fibrous histiocytomas share very similar comparative genomic hybridization imbalances: an analysis of a series of 27 leiomyosarcomas. Lab Invest 2001;81:211-5.

28. Ladanyi M, Bridge JA. Contribution of molecular genetic data to the classification of sarcomas. Hum Pathol 2000;31: 532-8.

29. Miyajima K, Tamiya S, Oda Y, Adachi T, Konomoto T, Toyoshiba $\mathrm{H}$, et al. Relative quantification of p53 and $\mathrm{mdm} 2$ gene expression in leiomyosarcoma; real-time semi-quantitative reverse transcription-polymerase chain reaction. Cancer Lett 2001;164:177-88.

30. Pilotti S, Della Torre G, Lavarino C, Di Palma S, Sozzi G, Minoletti F, et al. Distinct mdm2/p53 expression patterns in liposarcoma subgroups: implications for different pathogenetic mechanisms. J Pathol 1997;181:14-24.

31. Dei Tos AP, Doglioni C, Piccinin S, Sciot R, Furlanetto A, Boiocchi $\mathrm{M}$, et al. Coordinated expression and amplification of the MDM2, CDK4, and HMGI-C genes in atypical lipomatous tumours. J Pathol 2000;190:531-6.

32. Nilbert M, Rydholm A, Willen H, Mitelman F, Mandahl N. MDM2 gene amplification correlates with ring chromosomes in soft tissue tumors. Genes Chromosomes Cancer 1994;9:261-5.

33. Nakayama T, Toguchida J, Wadayama B, Kanoe H, Kotoura Y, Sasaki MS. MDM2 gene amplification in bone and softtissue tumors: association with tumor progression in differentiated adipose-tissue tumors. Int J Cancer 1995;64:342-6.

34. Reid AH, Tsai MM, Venzon DJ, Wright CF, Lack EE, O'Leary TJ. MDM2 amplification, P53 mutation, and accumulation of the P53 gene product in malignant fibrous histiocytoma. Diagn Mol Pathol 1996;5:65-73. 